\title{
VOLTERRA INTEGRAL EQUATIONS WITH UNKNOWN DELAY TIME
}

\author{
Yuri Yatsenko
}

\begin{abstract}
Systems of Volterra integral equations with unknown lower limits of integration describe some models of developing economics. The integral equation with unknown upper limit of integration arises in dual problems for optimal control by such models. The solvability of these equations is studied in the present paper.
\end{abstract}

\section{Introduction}

Volterra integral equations (VIEs) of the form

$$
x(t)=\int_{t-T}^{t} K(\tau, t, x(\tau)) d \tau, \quad x(\tau) \in \mathbb{R}^{n},
$$

are used extensively in the analysis of dynamical systems. The widening range of applications recently has led to studying equations (1) whose delay is an unknown function of time, i.e., $T=T(t)>0$.

The integral equations examined below arise in the class of economic-mathematical models with controlled liquidation (winding-up, elimination) of obsolete production units (PUs) [9-13] under technological change [5, 18]. Their main novelty is the introduction of a new endogenous function $z(t)$ which is the time limit for the use of production units, i.e., the units created before the time $z(t)$ are not used at the current time $t$. These models are described by the systems of nonlinear Volterra-type equations (1) with unknown function $z(t)=t-T(t)$ in the lower limit of integration. Such VIEs fall among the equations with unknown deviating arguments (especially with retarded ones). This paper deals with the theory of such equations.

The paper is organized as follows. In Section 2, we prove sufficient conditions for existence and uniqueness of the solutions of the system of two nonlinear VIEs with one unknown lower limit of integration. In Sections 3 and 4, the analogous results are established for a similar system of the first kind as well as for the system of $2 n$ VIE s with $n$ unknown lower integral limits, $n>1$. In Section 5 , the integro-functional equation of non-Volterra type with unknown upper integral limit (with unknown forestalling argument) is studied, and the existence, uniqueness, and asymptotics of its solutions are investigated. It arises in the optimization problems for the VIE s considered here. Examples of exact solutions are given in all sections. In the last section, we briefly describe some applications of VIE s with unknown delay time.

\section{The investigation of VIE $s$ with unknown lower limit of integration}

If the variable lower limits of integration in VIE s are given functions, then the methods for investigating such equations are analogous [16] to the well-developed methods for

Received March 9, 1994 , revised April 21, 1995.

1991 Mathematics Subject Classification. 45L10.

Key words and phrases: integral equations, dynamical systems. 
ordinary VIEs $[6,7]$. The presence of an unknown limit, if only one, in the VIE determines the novelty of the research on the principal theoretical problems (existence, uniqueness, stability of solutions, etc.) brought about by these equations [10-12, 22].

Let us consider the system of nonlinear Volterra integral equations in the unknown $x(t)$, where $z(t), t \in\left[t_{0}, T\right), t_{0}<T \leq \infty$,

$$
\begin{aligned}
& x(t)=\int_{z(t)}^{t} f(\tau, t, x(\tau)) d \tau \\
& \int_{z(t)}^{t} \varphi(\tau, t, x(\tau)) d \tau=p(t),
\end{aligned}
$$

with the initial conditions

$$
z\left(t_{0}\right)=z_{0}<t_{0}, \quad x(\tau) \equiv x_{0}(\tau), \quad \tau \in\left(-\infty, t_{0}\right] .
$$

In view of automatic control theory, the expressions (2)-(3) represent the integral model of a nonlinear dynamical system with entry $x(t)$ and unknown delay duration $t-z(t)$.

Assume that the given functions $f(\tau, t, x), \varphi(\tau, t, x), p(t), x_{0}(\tau)$ are continuous, non-negative at $\tau \in(-\infty, T), t \in\left[t_{0}, T\right), x \in[0, \infty)$, and satisfy (2)-(3) at $t=t_{0}$.

Theorem 1. Assume

(1) $f(\tau, t, x)$ and $\varphi(\tau, t, x)$ are positive for $\tau \in(-\infty, T), t \in\left[t_{0}, T\right), x \in(0, \infty)$;

(2) the integral $\int_{-\infty}^{t_{0}} \varphi\left(\tau, t, x_{0}(\tau)\right) d \tau$ diverges, $t \in\left[t_{0}, T\right)$; and

(3) $f(\tau, t, x), \varphi(\tau, t, x)$, and $p(t)$ satisfy the Lipschitz condition in $x, t$, and $f(\tau, t, x) / \varphi(\tau, t, x)$ is bounded, $\tau \in(-\infty, T), t \in\left[t_{0}, T\right), x \in[0, \infty)$.

Then the system of equations (2)-(3) has a unique solution $x \in \mathcal{C}\left[t_{0}, T\right), z \in \mathcal{C}\left[t_{0}, T\right)$, such that $x(t)>0$ and $z(t)<t$.

Proof. The equations (2)-(3) can be represented in the form

$$
\begin{gathered}
x(t)=\int_{t_{0}}^{t} f(\tau, t, x(\tau)) d \tau+\int_{z(t)}^{t_{0}} f\left(\tau, t, x_{0}(\tau)\right) d \tau \\
\int_{z(t)}^{t_{0}} \varphi\left(\tau, t, x_{0}(\tau)\right) d \tau=p(t)-\int_{t_{0}}^{t} \varphi(\tau, t, x(\tau)) d \tau \stackrel{\mathrm{df}}{=} c(t),
\end{gathered}
$$

$t \in\left[t_{0}, t_{1}\right], t_{1} \leq T$, where the time $t_{1}$ will be determined later. Introduce the functions

$$
\left.F(z, t)=\int_{z}^{t_{0}} f\left(\tau, t, x_{0}(\tau)\right) d \tau, \quad \Phi(z, t)=\int_{z}^{t_{0}} \varphi\left(\tau, t, x_{0}(\tau)\right)\right) d \tau,
$$

which are defined for $-\infty<z \leq t_{0}$. Under the conditions of the theorem, $\Phi(z, t)$ is strictly increasing in $z$ and has the unique inverse (in $z) \Phi^{-1}(c, t)$, which is defined for $c \in[0, \infty)$. Let $S(c, t)=F\left[\Phi^{-1}(c, t), t\right]$. If $c(t) \geq 0$, then one can determine the unknown $z$ from (6) since $z(t)=\Phi^{-1}(c, t)$, and substitute it into (5). Thus, the system of equations (5)-(6) is equivalent to the nonlinear Volterra integral equation

$$
x(t)=A x \equiv \int_{t_{0}}^{t} f(\tau, t, x(\tau)) d \tau+S\left[p(t)-\int_{t_{0}}^{t} f(\tau, t, x(\tau)) d \tau, t\right],
$$

if $c(t) \geq 0$, i.e., if the condition

$$
p(t) \geq \int_{t_{0}}^{t} \varphi(\tau, t, x(\tau)) d \tau
$$


is valid. Denote the set of continuous functions $\left\{x(t), t \in\left[t_{0}, t_{1}\right]: 0 \leq x(t) \leq x_{0}\left(t_{0}\right)+\right.$ $d=D_{0}, d=$ const $\left.>0\right\}$ by $\Omega_{d}$, and introduce the norm

$$
\|f\|=\max _{\substack{\tau, t \in\left[t_{0}, t_{1}\right] \\ x \in\left[0, D_{0}\right]}}|f(\tau, t, x)|
$$

Condition (9) is fulfilled if $x \in \Omega_{d}$ and

$$
t_{1}-t_{0} \leq \min _{\left[t_{0}, t_{1}\right]} \frac{p(t)}{\|\varphi\|}
$$

$\left(\right.$ then $\left.p(t) \geq \min _{\left[t_{0}, t_{1}\right]} p(t) \geq\|\varphi\|\left(t_{1}-t_{0}\right) \geq \int_{t_{0}}^{t} \varphi(\tau, t, x(\tau)) d \tau\right)$.

The function $S(c, t)$ satisfies a Lipschitz condition with constant

$$
L_{s}=\max |f(\tau, t, x) / \varphi(\tau, t, x)| .
$$

Denote the Lipschitz constants of the functions $f(\tau, t, x), \varphi(\tau, t, x)$, and $p(t)$ by $L_{f}^{x}, L_{f}^{t}$, $L_{\varphi}^{x}, L_{\varphi}^{t}$, and $L_{p}$. It follows from the second condition of the theorem and (9) that $0 \leq t_{0}-z(t) \leq C_{z}, t \in\left[t_{0}, t_{1}\right]$, where $C_{z}$ is some constant.

We now estimate the difference $x^{*}(t)-x_{0}\left(t_{0}\right), x^{*}=A x$. After a standard transformation, we find

$$
\begin{aligned}
x^{*}(t)-x_{0}\left(t_{0}\right) & \leq\left[\|f\|+L_{s}\left(L_{p}+\|\varphi\|\right)+\left(L_{f}^{t}+L_{s} L_{\varphi}^{t}\right) C_{z}\right]\left(t-t_{0}\right) \\
& \stackrel{\mathrm{df}}{=} C_{A}\left(t-t_{0}\right) .
\end{aligned}
$$

Recalling that $A x \geq 0$, we find that the operator $A$ is invariant with respect to the set $\Omega_{d} \subset \mathcal{C}\left[t_{0}, t_{1}\right]$ for

$$
t_{1}-t_{0} \leq \frac{d}{C_{A}}
$$

Finally, it may be proved that

$$
\left\|A x_{1}-A x_{2}\right\|_{\left[t_{0}, t_{1}\right]} \leq\left(L_{f}^{x}+L_{s} L_{\varphi}^{x}\right)\left(t_{1}-t_{0}\right)\left\|x_{1}-x_{2}\right\|_{\left[t_{0}, t_{1}\right]}
$$

is valid for $x_{1}, x_{2} \in \Omega_{d}$. Hence, the operator $A$ is contracting provided that

$$
t_{1}-t_{0}<\frac{1}{L_{f}^{x}+L_{s} L_{\varphi}^{x}}
$$

Choose the time $t_{1}$ such that the inequalities (10), (12), and (13) are fulfilled. Then the equation (8) and system (5)-(6) have a unique solution $x \in \mathcal{C}\left[t_{0}, t_{1}\right]$ by virtue of the Contraction Mapping Principle [7]. Furthermore, $x(t) \geq 0, t \in\left[t_{0}, t_{1}\right]$, in view of the positiveness of the function $S$ in (8). The unique solution $z(t)<t_{0}, t \in\left[t_{0}, t_{1}\right]$, is determined by (6).

Since we now may take $x(t)=x_{0}(\tau)$ to be given on $\left(-\infty, t_{1}\right]$, we prove the existence and uniqueness of the solution $x(t), t \in\left[t_{1}, t_{2}\right], t_{1}<t_{2}<T$, etc. We shall show that any finite interval $\left[t_{0}, T_{1}\right], T_{1}<T$, may be gone through by a finite number of steps $t_{i+1}-t_{i}$ determined by the inequalities (10), (12), and (13). Indeed, the constant $\delta_{1}=1 /\left(L_{f}^{x}+L_{s} L_{\varphi}^{x}\right)$ in the right-hand side of (13) is the same for all intervals $\left[t_{i}, t_{i+1}\right]$. Next, in view of the second condition of the theorem, it follows from (5)-(6) that the constant $C_{z}$ may be chosen as $C_{z}=\max _{\left[t_{0}, T_{1}\right]} \Phi^{-1}\left(\max _{\left[t_{0}, T_{1}\right]} p(t), t\right)+t_{0}$, independent of the interval number $i$. Hence, the constant $\delta_{2}=d / C_{A}$ in (12) also may be chosen independent of $i$. Finally, if we take $\|\varphi\|$ as the norm for $\tau, t \in\left[t_{0}, T_{1}\right]$, then the constant $\delta_{3}=\min _{\left[t_{0}, T_{1}\right]} p(t) /\|\varphi\|$ does not depend on $i$. Thus, $t_{i+1}-t_{i} \geq \delta=$ $\min \left(\delta_{1}, \delta_{2}, \delta_{3}\right)$ for any $i$; hence, the unique solution $x(t)$ exists on any finite interval $\left[t_{0}, T_{1}\right], T_{1}<T$.

Thus, the theorem is proved. 
Remark 1. When studying the problems of optimal control by equations of the form (2)-(3), it often is required to study wider classes of functions $f$ and $\varphi$ measurable with respect to $\tau$ [10-12], but the result of Theorem 1 holds true [22].

Example 1. Let $f(\tau, t, x)=b_{0} e^{c_{0}(\tau-t)} x^{d_{0}}, \varphi(\tau, t, x)=b_{1} e^{c:(\tau-t)} x^{d_{1}}, 0<d_{i}<1$, $b_{i}, c_{i}>0, i=0,1$ (the so-called Cobb-Douglas production functions with exponential technological change and exponential decay $[5,13,18]), p(\cdot) \equiv p, x_{0}(\cdot) \equiv x_{0}, z\left(t_{0}\right)=$ $t_{0}-D$, where the constants $p, D$, and $x_{0}$ satisfy the system of nonlinear equations

$$
\begin{aligned}
& 1-e^{-c_{0} D}=\frac{c_{0} x_{0}^{1-d_{0}}}{b_{0}}, \\
& 1-e^{-c_{1} D}=\frac{c_{1} p x_{0}^{-d_{1}}}{b_{1}} .
\end{aligned}
$$

Then the solution of equations (2)-(3) is

$$
z(t) \equiv t-D, \quad x(t) \equiv x_{0}, \quad t \in\left[t_{0}, T\right),
$$

When $c_{0}, c_{1} \ll 1, \quad D=\left[\left(p / b_{1}\right)^{1-d_{0}} b_{0}^{-d_{1}}\right]^{1 /\left(1-d_{0}+d_{1}\right)}+o\left(c_{0}, c_{1}\right)$,

$$
x_{0}=\left(\frac{p b_{0}}{b_{1}}\right)^{1 /\left(1-d_{0}+d_{1}\right)}+o\left(c_{0}, c_{1}\right) \text {. }
$$

In applied problems, the prehistory $x_{0}(\tau)$ of the process $x(t)$ is usually given for some bounded interval $\left[\tau_{0}, t_{0}\right],-\infty<\tau_{0}<t_{0}$. Then, in view of Theorem 1 , the solution of equations (2)-(3) can cease to exist. The following theorem provides the conditions under which the value $z(t)$ belongs to the interval $\left[\tau_{0}, t\right]$; hence, the solution $(x(\cdot), z(\cdot))$ exists.

Theorem 2. If the conditions of Theorem 1 are fulfilled, the functions $f(\tau, t, x)$ and $\varphi(\tau, t, x)$ are differentiable with respect to $x$, then $\tau_{0} \leq z(t) \leq t, t \in\left[t_{0}, T\right)$, if and only if

$$
p(t) \leq \bar{p}(t)=\int_{\tau_{0}}^{t} \varphi(\tau, t, \bar{x}(\tau)) d \tau .
$$

where $\bar{x}$ is determined by the equation

$$
\bar{x}(t)=\int_{t_{0}}^{t} f(\tau, t, \bar{x}(\tau)) d \tau+\int_{\tau_{0}}^{t_{0}} f\left(\tau, t, x_{0}(\tau)\right) d \tau .
$$

In this case, $x(t) \leq \bar{x}(t), t \in\left[t_{0}, T\right)$.

Proof. Varying the system (2)-(3), we obtain the system of linear integral Volterra equations with respect to the variations $\delta x(t), \delta z(t), \delta p(t), t \in\left[t_{0}, T\right)$, of the unknown solutions $x(\cdot), z(\cdot)$, and the given function $p(\cdot)$. Using well-known properties of Volterra operators [7], we obtain from this system that $\delta x(\cdot) \geq 0$ and $\delta z(\cdot) \leq 0$ for $\delta p(\cdot) \geq 0$. In the case of the function $p(\cdot)$ given by (15), this implies the required assertion. The theorem is proved.

The existence and uniqueness of solutions of nonlinear integral equations distinct from (10)-(12) with the unknowns in the limits of integration are studied in [14]. 


\section{The VIEs of the first kind with unknown limit of integration}

Let us consider the system of VIEs of the first kind in the unknowns $y(t), z(t), t \in$ $\left[t_{0}, T\right), t_{0}<T \leq \infty$

$$
\begin{aligned}
& \int_{z(t)}^{t} \varphi(\tau, t, y(\tau)) d \tau=p(t) \\
& \int_{z(t)}^{t} \psi(\tau, t, y(\tau)) d \tau=q(t)
\end{aligned}
$$

with the initial conditions

$$
z\left(t_{0}\right)=z_{0}<t_{0}, \quad y(\tau) \equiv y_{0}(\tau), \quad \tau \in\left(-\infty, t_{0}\right] .
$$

Suppose that the given functions $\varphi(\tau, t, y), \psi(\tau, t, y), p(t), q(t)$, and $y_{0}(\tau)$ are continuously differentiable with respect to $t$ and $y$, Lipschitz continuous with respect to $\tau$, positive, and satisfy (16)-(18) at $t=t_{0}$.

Theorem 3. Assume

(1) $\varphi_{t}^{\prime}, \psi_{t}^{\prime}, p^{\prime}, q^{\prime}$ satisfy Lipschitz conditions in $y$ and $t, \tau \in(-\infty, T), t \in\left[t_{0}, T\right)$, $y \in[0, \infty)$

(2) $\int_{-\infty}^{t_{0}} \varphi\left(\tau, t, y_{0}(\tau)\right) d \tau$ and $\int_{-\infty}^{t_{0}} \psi\left(\tau, t, y_{0}(\tau)\right) d \tau$ diverge, $t \in\left[t_{0}, T\right)$;

(3) $\varphi_{t}^{\prime}(\tau, t, y) / \varphi(\tau, t, y)$ and $\psi_{t}^{\prime}(\tau, t, y) / \varphi(\tau, t, y)$ are bounded, $\tau \in(-\infty, T), t \in$ $\left[t_{0}, T\right), y \in[0, \infty)$

(4) the inequality $\varphi_{y}^{\prime}(t, t, x)>\psi_{y}^{\prime}(t, t, x) k(\tau, t, y)$ holds where

$$
k(\tau, t, y)=\varphi(\tau, t, y) / \psi(\tau, t, y), \tau \in(-\infty, T), t \in\left[t_{0}, T\right), x, y \in[0, \infty) ;
$$

(5) for $u<\tau<t$, the inequalities $\varphi_{t}^{\prime}(\tau, t, x)<\psi_{t}^{\prime}(\tau, t, x) k(u, t, y)$ and $p^{\prime}(t)>$ $q^{\prime}(t) k(\tau, t, y)$ hold, $u, \tau \in(-\infty, T), t \in\left[t_{0}, T\right), x, y \in[0, \infty) ;$ and

(6) $\varphi(t, t, 0) \geq \Psi(t, t, 0) k(\tau, t, y), \tau \in(-\infty, T), t \in\left[t_{0}, T\right), y \in[0, \infty)$.

Then the system of equations (16)-(17) has the unique solution $y \in \mathcal{C}\left[t_{0}, T\right), z \in$ $\mathcal{C}\left[t_{0}, T\right)$, such that $y>0$ and $z(t)<t$.

Proof. By differentiating (16)-(17) and eliminating $z^{\prime}(t)$, we obtain an integral equation of the second kind,

$$
\Omega(y(t), z(t), t)=\int_{z(t)}^{t} \chi(y(\tau), z(t), \tau, t) d \tau+R(z(t), t),
$$

$t \in\left[t_{0}, t_{1}\right], t_{1}$ satisfies (10), where

$$
\begin{aligned}
\Omega(y, z, t) & =\varphi(t, t, y) \psi\left(z, t, y_{0}(z)\right)-\psi(t, t, y) \varphi\left(z, t, y_{0}(z)\right), \\
\chi(y, z, \tau, t) & =\psi_{t}^{\prime}(\tau, t, y) \varphi\left(z, t, y_{0}(z)\right)-\varphi_{t}^{\prime}(\tau, t, y) \psi\left(z, t, y_{0}(z)\right), \\
R(z, t) & =p^{\prime}(t) \psi\left(z, t, y_{0}(z)\right)-q^{\prime}(t) \varphi\left(z, t, y_{0}(z)\right) .
\end{aligned}
$$

Equation (19) and one of the equations (16)-(17) form a system of nonlinear equations which is analogous to the system (2)-(3). In view of the fourth condition of the theorem, the function $\Omega$ has the inverse function $\Omega^{-1}$ in $y$ and, thus,

$$
y(t)=\Omega^{-1}\left[\int_{z(t)}^{t} \chi(y(\tau), z(t), \tau, t) d \tau+R(z(t), t), t\right] .
$$

Reasoning as in the proof of Theorem 1, we reduce the system (2), (20) on the interval $\left[t_{0}, t_{1}\right], t_{1} \leq T$, to one nonlinear Volterra equation which is solvable because of the Contraction Mapping Principle and the first three conditions of the theorem. 
The fifth condition ensures positiveness of the right-hand side of equation (19), whence, in view of the last condition, positiveness of $y(\cdot)$ follows. The theorem is proved.

The fourth and fifth conditions of Theorem 3 seem to be very restrictive, but they are valid in many cases. For example, in the two-sector integral model of economic renovation [22, 24], $\varphi(\tau, t, y)=\alpha(\tau, t) y, \psi(\tau, t, y)=\beta(\tau, t)[1-y]$, hence, the fourth condition holds true for all $\alpha, \beta>0$.

Another important case arises when $k(\tau, t, y)$ does not depend on $y$. Then the mentioned conditions are more easily verified; in particular, the fifth one leads to the inequality

$$
\frac{\varphi_{t}^{\prime}(\tau, t, y)}{\psi_{t}^{\prime}(\tau, t, y)}<k(u, t)<\frac{p^{\prime}(t)}{q^{\prime}(t)} \quad \text { if } \psi_{t}^{\prime}, q^{\prime}>0 .
$$

Example 2. Let $\varphi(\tau, t, y)=c \tau y^{d}, c>0,0<d<1, \psi(\tau, t, y)=y^{d}, p(t)=c t-c D / 2$, $q(\cdot) \equiv 1, y_{0}(\cdot) \equiv y_{0}, z\left(t_{0}\right)=t_{0}-D$ where $D=y_{0}^{-d}$. Then the solution of the equations $(16)-(17)$ is

$$
z(t) \equiv t-D, \quad y(t) \equiv y_{0}, \quad t \in\left[t_{0}, T\right) .
$$

It should be noted that Theorem 3 represents one version of the sufficient conditions for the existence and uniqueness of the solution for the equations (16)-(17). The derivation of the necessary and sufficient conditions runs into great difficulties.

\section{VIEs with several unknown lower limits of integration}

We shall restrict ourselves to the system of $2 n$ VIEs of the second kind with linear integrands in the unknowns given by $x_{1}(t), \ldots, x_{n}(t), z_{1}(t), \ldots, z_{n}(t), t \in\left[t_{0}, T\right), t_{0}<$ $T \leq \infty$,

$$
\begin{gathered}
x_{i}(t)=\sum_{j=1}^{n} \int_{z_{j}(t)}^{t} K_{i j}(\tau, t) x_{j}(\tau) d \tau \\
\sum_{j=1}^{n} \int_{z_{j}(t)}^{t} Q_{i j}(\tau, t) x_{j}(\tau) d \tau=p_{i}(t), \quad i=1, \ldots, n,
\end{gathered}
$$

with the initial conditions

$$
z_{j}\left(t_{0}\right)=z_{j}^{0}<t_{0}, \quad x_{j}(\tau) \equiv x_{j}^{0}(\tau), \quad \tau \in\left(-\infty, t_{0}\right], \quad j=1, \ldots, n .
$$

In contrast to the previous cases, the positiveness of the given functions proved insufficient for the inequalities $z_{j}(t)<t$ to hold when several $z_{j}(t)$ are sought, and therefore more restrictive relations between $p_{j}(t)$ and $Q_{i j}(\tau, t)$ are required. Let us illustrate these for the case with a triangular matrix $\left\{Q_{i j}\right\}$.

Theorem 4. Assume

(1) the functions $K_{i j}(\tau, t), p_{i}(t), x_{i}^{0}(\tau), Q_{i i}(\tau, t)$ are positive, $Q_{i k}(\tau, t) \geq 0$ for $k<$ $i, Q_{i k}(\tau, t)=0$ for $k>i, \tau \in(-\infty, T), t \in\left[t_{0}, T\right)$;

(2) $K_{i j}(\tau, t), Q_{i j}(\tau, t), p_{i}(t), x_{i}^{0}(\tau)$ are continuous with respect to $\tau$ and satisfy $a$ Lipschitz condition in $t, i, j=1, \ldots, n, \tau \in(-\infty, T), t \in\left[t_{0}, T\right)$;

(3) the integrals $\int_{-\infty}^{t_{0}} Q_{i i}(\tau, t) x_{0}^{i}(\tau) d \tau$ diverge, $i=1, \ldots, n, t \in\left[t_{0}, T\right)$; and

(4) for each $i=1, \ldots, n-1$ the inequality $p_{i+1}(t) / p_{i}(t)>Q_{i+1 i}\left(u_{1}, t\right) / Q_{i i}\left(u_{1}, t\right) \geq$ $Q_{i+1 i-1}\left(u_{2}, t\right) / Q_{i i-1}\left(u_{2}, t\right) \geq \cdots \geq Q_{i+1 i}\left(u_{i}, t\right) / Q_{i 1}\left(u_{i}, t\right)$ is fulfilled at $u_{1}$, $u_{2}, \ldots, u_{i} \in \mathbb{R}, \tau \in(-\infty, T), t \in\left[t_{0}, T\right)$.

Then the system (21)-(23) has the unique solution $x_{i}, z_{i} \in \mathcal{C}\left[t_{0}, T\right), i=1, \ldots, n$, such that $x_{i}(t)>0$ and $z_{i}(t)<t$. 
Proof. The proof is analogous to that of Theorem 1. The first and third conditions of the theorem ensure that the equations (22) have a unique solution $z_{i}(\cdot), i=1, \ldots, n$, at the given $x_{i}(t)>0, i=1, \ldots, n$. The last condition assures that $z_{i}(t)<t$, $t \in\left[t_{0}, T\right)$.

Example 3. Let $K_{i j}(\tau, t)=b_{i j} e^{c_{i j}(\tau-t)}, Q_{i i}(\tau, t)=\beta_{i} e^{\gamma_{i}(\tau-t)}, Q_{i j}(\tau, t) \equiv 0$ at $i \neq j$, $b_{i j}, c_{i j}, \beta_{i}, \gamma_{i}>0, p_{i}(\cdot) \equiv p_{i}, x_{j}^{0}(\cdot) \equiv x_{j}^{0}, z_{i}\left(t_{0}\right)=t_{0}-D_{i}, i, j=1, \ldots, n$, where the constants $p_{i}, D_{i}$, and $x_{i}^{0}$ satisfy the system of $2 n$ nonlinear equations

$$
\begin{gathered}
x_{i}^{0}=\sum_{j} \frac{b_{i j}\left(1-e^{-c_{i j} D_{j}}\right)}{c_{i j}} x_{j}^{0}, \\
p_{i}=\frac{x_{i}^{0} \beta_{i}\left(1-e^{-\gamma_{i} D_{i}}\right)}{\gamma_{i}}, \quad i=1, \ldots, n .
\end{gathered}
$$

Then the solution of the equations (21)-(23) is

$$
z_{i}(t) \equiv t-D_{i}, \quad x_{i}(t) \equiv x_{i}^{0}, \quad i=1, \ldots, n, t \in\left[t_{0}, T\right) .
$$

If $c_{i j}, \gamma_{i} \ll 1$, then

$$
\begin{gathered}
D_{i}=\frac{p_{i}\left(\sum_{j} b_{i j} p_{j} / \beta_{j}\right)^{-1}}{\beta_{i}}+o\left(c_{i j}, \gamma_{i}\right), \\
x_{i}^{0}=\sum_{j} \frac{b_{i j} p_{j}}{\beta_{j}}+o\left(c_{i j}, \gamma_{i}\right), \quad i=1, \ldots, n .
\end{gathered}
$$

\section{Analysis of integral-functional equation with unknown upper limit of integration}

Optimal control problems usually evoke great interest among those engaged in economic-mathematical simulation $[8,15]$, which is displayed by investigations into the class of VIE s under discussion [10-12, 22]. When we researched the optimal control problems using the VIEs considered here the integral-functional equation of nonVolterra type for the unknown function $z(t), t \in\left[t_{0}, \infty\right)$

$$
\int_{t}^{z^{-1}(t)} \rho(\tau)[\beta(t, \tau)-\beta(z(\tau), \operatorname{tau})] d \tau=\rho(t) \gamma(t),
$$

arises $[2,12,22,24]$ where $z^{-1}(\cdot)$ is the inverse function of $z(\cdot)$.

This equation plays an important role in the qualitative analysis of the solutions of optimal control problems. It has a clear economic interpretation, namely, the lefthand side of (24) describes a rollover estimate of efficiency - the incremental output produced by one new capacity unit (CU) during its unknown future lifetime $\left[t, z^{-1}(t)\right]$, compared with the output of the oldest existing CU. In view of (24), this value must be equal to the cost $\gamma(t)$ of a new CU. The function $t-z(t)$ is the optimal lifetime of CUs.

In accordance with the economic interpretation of (24), we shall restrict ourselves to the case of monotone continuously differentiable solutions $z(t): z(t)<t, t \in\left[t_{0}, \infty\right)$. The study of (24) is carried out in analogy to that in the case $\rho \equiv 1[22]$.

Suppose the $\rho(t)=e^{-d t}, d>0, \beta(\tau, t) \equiv \beta(\tau)$. The functions $\beta(\tau), \gamma(t)$ are twice differentiable and $\beta>0$. Under these conditions, (24) is of the form

$$
\int_{t}^{z^{-1}(t)}[\beta(t)-\beta(z(\tau))] e^{-d \tau} d \tau=e^{-d t} \gamma(t) .
$$


By differentiating (25), we obtain the recurrence relation

$$
\beta(z(t))-\beta(t)+\beta(t) \frac{1-e^{-d\left(z^{-1}(t)-t\right)}}{d}=\gamma(t)-d \gamma(t)
$$

which connects the values $z(t)$ and $z^{-1}(t)$.

We will analyze the conditions for given functions $\beta$ and $\gamma$ under which (25) can have a solution with $z_{0}<z(t)<t, z^{\prime}(t)<0$. Suppose $z(u) \equiv z(t), u>t$. Then $z^{-1}(u)=\infty, u>z^{-1}(t)$, and $\beta(z(t))-\beta(t)=d \gamma(t)$ follows from (26). Thus, for the solution $z(t), z^{\prime}(t)>0$, to exist, it is necessary that $\beta(z(t))-\beta(t)>d \gamma(t)$. Let

$$
\frac{\gamma(t)}{\beta(t)} \underset{t \rightarrow \infty}{\longrightarrow} 0
$$

Then $z(t)$ can tend to $\infty$ when $t \rightarrow \infty$. Suppose that the condition (27) is satisfied in the following.

Lemma 1. Let equation (25) have a solution $z \in \mathcal{C}^{1}\left[t_{0}, T\right]$ and another solution $z+$ $\delta z \in \mathcal{C}^{1}\left[t_{0}, T\right], \delta z(t)=o(z(t))$, which is close to the first. If $z^{\prime}\left(z^{-1}(t)\right)<L(z(t))$ where

$$
L(z)=e^{-d\left(z^{-1}(t)-t\right)} \frac{\beta(t)}{\beta(z(t))},
$$

then $|\delta z(t)|>\left|\delta z\left(z^{-1}(t)\right)\right|$ (the variation $\delta z(t)$ of $z(t)$ is decreasing) and conversely. And if $z^{\prime}\left(z^{-1}(t)\right)<\sqrt{L(z(t))}$, then $\left|\delta z^{\prime}(t)\right|>\left|\delta z^{\prime}\left(z^{-1}(t)\right)\right|$ (the variation $\delta z^{\prime}(t)$ of the derivative $z^{\prime}(t)$ is decreasing) and conversely.

Proof. The proof of Lemma 1 in the case $d=0$ was shown in [22]. In the general case, the proof proceeds in a similar way and is based upon varying (25) with respect to $z(\cdot)$, varying $(26)$ with respect to $z^{\prime}(\cdot)$, and the use of the equality

$$
\delta z^{-1}(t)=\frac{\delta z\left(z^{-1}(t)\right)}{z\left(z^{-1}(t)\right)}+o\left(\delta z^{-1}(t)\right)
$$

Lemma 1 allows us to show the existence and the uniqueness of the solution of equation (25) on the unbounded interval $\left[t_{0}, \infty\right)$ in some cases as well as the asymptotic behavior of solutions for bounded intervals $\left[t_{0}, T\right], T-t_{0} \gg 1$.

In [22], when $d=0$, it was shown that for existence of a continuous solutions $z(t), t \in\left[t_{0}, T\right], T \gg t_{0}$, of $(25)$, the given functions $\beta(\cdot), \gamma(\cdot)$, and the solution $z(\cdot)$ themselves must satisfy rather strong conditions, and this solution was constructed in the cases of exponential, power, and logarithmic functions $\beta(\tau)$. We shall restrict ourselves to the following cases.

Theorem 5. Let $\beta(\tau)=c_{1} \tau+c_{2}, \gamma(t)=\gamma \equiv$ const, $c_{1}, \gamma>0$. Then (25) has the unique solution $\tilde{z}(t)=t-A, t \in\left[t_{0}, \infty\right)$, and some set of solutions $z(t), z^{\prime}(t)>0$, for any interval $\left[t_{0}, T\right]$. In the case where $T \rightarrow \infty$, all solutions $z(\cdot)$ approach the solution $\tilde{z}(\cdot)$. The constant $A$ is determined by the nonlinear equation

$$
A-\frac{1-e^{-d A}}{d}=-\frac{d \gamma}{c_{1}}
$$

$\left(A \cong \sqrt{2 \gamma / c_{1}}+o(d)\right.$, when $\left.d \ll 1\right)$.

Proof. It is easily verified that (25) has the solution $\tilde{z}(t)=t-A$ for the infinite interval $\left[t_{0}, \infty\right)$ where the constant $A$ is determined by $(29)$. This fact can be checked directly. We shall show that this solution is unique. Indeed, in this case $L(z)=e^{-d A}<1$. 
Thus, if $z^{\prime}(\tau) \neq 1$ and $z^{\prime}(\tau)>\sqrt{e^{-d A}}$ at some point $\tau>t_{0}$, then according to Lemma 1, this results in increasing $\left|\delta z^{\prime}(t)\right|$ and $|\delta z(t)|$ when $t \rightarrow \infty$, violating the condition $z^{\prime}(t)>0$ and, hence, yields the discontinuities of $z^{-1}(t)$. The continuous solution $z(t), t \in\left[t_{0}, z^{-1}\left(t_{0}\right)\right]$, such that $z^{\prime}(t)<\sqrt{e^{-d A}}$ cannot be constructed. For this reason, the unique continuous solution of $(25)$ for the interval $\left[t_{0}, \infty\right)$ is of the form $z(t)=t-A$.

Let us construct the solutions of (25) for some interval $\left[t_{0}, T\right], T \gg t_{0}$. We may construct some continuously differentiable function $z(t)<t, z^{\prime}(t)>\sqrt{e^{-d A}}>e^{-d A}$, $t \in[z(T), T]$, which satisfies (25) and (26) at $t=z(T)$. The construction of such a function can be done in more than one way. Continuing $z(t)$ recursively from the right to the left by means of the formula (26), we get, in view of Lemma 1 , some solution $z(t)<t, z^{\prime}(t)>0$ on the entire interval $\left[t_{0}, T\right]$. At the same time, any solution $z(t)$, $t \in\left[t_{0}, T\right]$, of $(25)$ tends to $\tilde{z}(\cdot)$ for $T \rightarrow \infty$, in view of Lemma 1 . The theorem is proved.

Example 4. Let $\beta(\tau)=\tau, t_{0}=1, \gamma=1, d=0.01$. Then $A \approx \sqrt{2}$ and the solution of $(25)$ is $\tilde{z}(t)=t-A, t \in[1, \infty)$.

Theorem 6. Let $\beta(\tau)=e^{c \tau}, \gamma(t)=b e^{s t}, c, b>0, s<c<d$. Then (25) has some set of solutions $z(t), z^{\prime}(t)>0$, for any interval $\left[t_{0}, T\right]$. In the case where $T \rightarrow \infty$, all these solutions tend to the unique solution $\tilde{z}(t), \tilde{z}^{\prime}(t)>0$, of $(25)$ on the interval $\left[t_{0}, \infty\right)$.

For $s<c, t-\tilde{z}(t) \rightarrow 0$ as $t \rightarrow \infty$, and $\tilde{z}(t) \equiv t-B$ at $s=c$, where the constant $B$ is determined by the nonlinear equation

$$
c e^{-d B}-d e^{-c B}=(b d-1)(d-c)
$$

$(B \cong \sqrt{2 b / c}+o(d)$, when $d \ll 1)$.

Proof. When $s=c$, substituting $z(t) \equiv t-B$ into (25) readily shows that $z(t)$ is a solution of (25), and we get (30) for defining $B$. In this case, $L(z)=e^{(c-d) B}$ is less than 1.

When $s<c<d$, according to (26), we have $\Delta(t)=t-z(t) \rightarrow 0$ for $t \rightarrow \infty$. Let us study the limiting behavior of the function $\Delta(t)$ when $t \rightarrow \infty$ (and $|\Delta(t)|$ is small). Let the time $t_{1}$ be fixed. Denote $\Delta\left(t_{1}\right)=\Delta_{1}, \Delta\left(z^{-1}\left(t_{1}\right)\right)=\Delta_{2}$, and let $\Delta(t)=\Delta_{1}+\varepsilon\left(t-t_{1}\right)+o\left(\Delta_{1}^{2}\right)$. Then B $=1-\Delta_{1} / \Delta_{2}$. Substituting $\Delta(t)$ into (25) and calculating the integral, we get

$$
\frac{1-e^{-d \Delta_{2}}}{d}+\frac{1}{d-c \Delta_{1} / \Delta_{2}}\left(e^{-d \Delta_{2}}-e^{-c \Delta_{1}}\right)+o\left(\Delta_{1}^{2}\right)=b e^{(s-c) t} .
$$

Using expansions of the left-hand side functions, we obtain the equation

$$
\frac{c \Delta_{1} \Delta_{2}}{2}+o\left(\Delta_{1}^{2}\right)=b e^{(s-c) t}
$$

which when substituted into (31) leads to a relation between $\Delta_{1}$ and $\Delta_{2}$

$$
d \Delta_{2}^{2}-\Delta_{2}(2+(d-s)) \Delta_{1}+2 \Delta_{1}-c \Delta_{1}^{2}+o\left(\Delta_{1}^{2}\right)=0 .
$$

A further study of the solution of this equation shows that $\Delta_{2}=\Delta_{1}+o(\Delta)$. Thus, when $t \rightarrow \infty, \Delta\left(z^{-1}(t)\right) / \Delta(t) \rightarrow 1$, and

$$
L(z)=e^{-d \Delta\left(z^{-1}(t)+c \Delta(t)\right)}=e^{-d \Delta_{2}+c \Delta_{1}} \rightarrow e^{-(d-c) \Delta_{1}+o\left(\Delta_{1}\right)}<1 .
$$

Now we may construct the solution of (25) in the same manner as in the previous case. If $z^{\prime}\left(z^{-1}(t)\right)>L(z(t))>\sqrt{L(z(t))}$ for any time $t$, then $|\delta z(t)|<\left|\delta z\left(z^{-1}(t)\right)\right|$ 
and $\left|\delta z^{\prime}(t)\right|<\left|\delta z^{\prime}\left(z^{-1}(t)\right)\right|$ because of Lemma 1. For this reason, we choose some time $T \gg t_{0}$ and construct the continuously differentiable function $\left.z(t)<t, z^{\prime}(t)\right\rangle$ $\sqrt{L(z)} \gg L(z), t \in\left[T, z^{-1}(T)\right]$, which satisfies (25) and (26) at $t=T$. Because of Lemma 1 , continuing $z(t)$ recursively from the right to the left by means of formula (26), we get some solution $z(t)<t, z^{\prime}(t)>0$ for the entire interval $\left[t_{0}, z^{-1}(T)\right]$. We shall obtain the solution $\tilde{z} \in \mathcal{C}^{1}\left[t_{0}, \infty\right)$ of $(25)$ if we let $T$ tend to $\infty$. In so doing, any solution of (25) satisfies $z(t) \rightarrow \tilde{z}(t)$ when $T, T-t \rightarrow \infty$. The theorem is proved.

In applications, the importance of (24) consists in the finding of some "effective" trajectories (so-called turnpike trajectories $[8,15]$ ) which are close to the solution of the optimization problem and have simpler structure. The turnpike theorems are among the most important in mathematical economics. The essence of turnpike properties is the statement that for long planned intervals, the optimal trajectory approaches the turnpike one, which is independent of the interval length and the optimization criterion. By means of the VIE s with unknown delay time considered here, the different turnpike theorems (in normal, strong, and strongest forms) have been established in $[22,24]$ for optimal trajectories $z(\cdot)$ of the economic system renovation (for the optimal capacity service periods).

In this connection, the study of general properties of turnpike trajectories defined by (24), if they exist, is useful. For this purpose, we represent the function $\beta$ in the form $\beta(\tau, t)=\beta_{1}(\tau) \beta_{2}(t)$ and emphasize the following four indicators $\beta_{1}^{\prime}(\tau), \beta_{2}(t), \gamma(t)$, and $\rho(t)$ which determine the behavior of turnpike trajectories $\tilde{a}(\cdot)$.

Theorem 7. Assume that $\beta_{1}^{\prime}(\tau)$ or $\beta_{2}(t)$ or $\rho(t)$ are increasing or that $\gamma(t)$ is decreasing, the other three indicators are constant, and there exists a solution $\tilde{z}(t), \tilde{z}^{\prime}(t)>0$, $t \in\left[t_{0}, \infty\right)$, of (24). Then the function $t-\tilde{z}(t)$ decreases as $t \rightarrow \infty$ and conversely. If all four indicators are constant, then $t-\tilde{z}(t) \equiv$ const, $t_{0} \leq t<\infty$.

Proof. The proof follows from the analysis of the properties of the (24) and its derivative.

\section{Some applications of VIEs with unknown delay time}

The necessity to apply integral equations in mathematical economics comes while considering the heterogeneity of economic factors ${ }^{*}$ such as fixed assets, production capacities, industrial equipment, labor force, etc. Such models take into account the materialized technological change $[5,18]$, which implies that new means of production are more efficient.

The macroeconomic models based on the above VIEs $[9-13,22]$ allow the investigation of the rational rates of technology renovation and dynamics of capacity lifetime. These problems are considered in correlation with other economic aspects (the presence of resources, production efficiency criteria, technological change rates, etc.).

These VIE s have other applications, which differ from macroeconomics. For example, they can describe the problems of equipment replacement in production systems $[4,17]$. The important problem of replacement models is to find the optimal time interval $T$ of replacement or repair [17]. For this purpose, the VIEs considered here make it possible to determine optimal $T$ as a dynamic function $T(t)$.

More generally, the VIEs with unknown delay time describe the models similar to those used in renewal theory $[1,20]$. This theory'arose from the study of so-called random "self-renewing" processes and has been a bright example of the importance of applying integral equations. The basic renewal equation is the linear VIE (1) with respect to the unknown $x(\cdot)$. Compared with renewal models, the VIEs considered 
here permit the investigation of essentially non-stationary processes with the variable renewal intervals $[z(t), t]$.

Similar integral models also arise in mathematical ecology, when the influence of the age structure and process prehistory upon population dynamics (hereditary effects) is considered ([3, 19, 21, 23] and others). For such problems, VIEs with unknown delay time may hold considerable promise. They incorporate a variable limiting age of individuals, a variation in the intensity of dying off and migration, etc.

Acknowledgment. The author expresses gratitude to the reviewer for his careful analysis of the manuscript and the useful notes which assisted in significant improvement of the paper.

\section{References}

1. G. K. Agrafiotis and M. Tsoukalas, Excess-time renewal theory with applications, J. Oper. Res. Soc., 41 (1990), 69-82.

2. D. D. Aistrahanov and Yu. P. Yatsenko, Approximate algorithms for modelling of economic systems renovation optimal terms, Cybernetics, 28 (1992), 168-173.

3. E. Aitdads and O. Arino, A non-Linear delay differential equation whose solutions are asymptotically sums of periodic functions, Funkcialaj Ekvacioj, 32 (1989), 81-89.

4. H. W. Block, W. S. Borges and T. H. Savits, A general age replacement model with minimal repair, Nav. Res. Logist., 35 (1988), 365-372.

5. M. Brown, On the Theory and Measurement of Technological Change, Cambridge, University Press, 1967.

6. H. Brunner and P. J. van der Houwen, The Numerical Solution of Volterra Equations, NorthHolland, Amsterdam, 1986.

7. T. A. Burton, Volterra Integral and Differential Equations, Academic Press, Orlando, Florida, 1983.

8. R. Dorfman, P. A. Samuelson, and R. Solow, Linear Programming and Economic Analysis, McGraw-Hill, 1958.

9. V. M. Glushkov, On a class of dynamical macroeconomic models, Upravljajushie systemy $i$ mashiny, (1977), 3-6 (in Russian).

10. V. M. Glushkov, V. V. Ivanov, and Yu. P. Yatsenko, Analytical investigation of a class of dynamical models. Parts I and II, Cybernetics, 16 (1980), 164-177; 18 (1982), 390-401.

11. 802-805 (in Russian).

12. V. V. Ivanov and Yu. P. Yatsenko, The problems of optimal control for the integral dynamical models with controllable prehistory, in Lecture Notes in Control and Information Sciences, Springer-Verlag, New York, 84 (1986), 350-357.

13. L. V. Kantorovich and V. I. Jianov, One-productional dynamical economic model describing change of fixed assets structure in presence of technical progress, Doklady Akad. Nauk SSSR, 211 (1973), 1280-1283 (in Russian).

14. M. Kwapisz and J. Turo, Existence and uniqueness of solution for some integral-functional equations, Rocz. RTM, Series 1, 23 (1983), 259-267.

15. K. Lancaster, Mathematical Economics, Macmillan, New York, 1968.

16. B. G. Pachpatte, On a nonlinear Volterra integral-functional equation, Funkcialaj Ekvacioj 26 (1983), 1-9.

17. S. -H. Sheu, A generalized block replacement policy with minimal repair and general random repair costs for a multi-unit system, J. Oper. Res. Soc. 42 (1991), 331-341.

18. R. Solow, Investment and technical progress, Mathematical Methods in the Social Science, Stanford Univ. Press, Stanford, 1960, pp. 21-40.

19. K. E. Swick, A nonlinear age-dependent model of single species population dynamics, SIAM J. Appl. Math., 32 (1977), 484-498.

20. H. C. Tijms, Stochastic Modelling and Analysis, Wiley, New York, 1986.

21. G. F. Webb, Theory of Nonlinear Age-Dependent Population Dynamics, Dekker, New York, 1985. 
22. Yu. P. Yatsenko, Integral Models of Systems with Controllable Memory, Naukova Dumka, Kiev, 1991 (in Russian).

23. _ Modelling of certain oscillating biological processes, Cybernetics, 14 (1978), 529-535.

24. Yu. Yatsenko and N. Hritonenko, Optimization in integral model of developing systems, Optimization, 31 (1994), 179-192.

Glushkov's Cybernetics Institute, 40 Glushkov Ave., 252207 Kiev, Ukraine 